\title{
The perturbation response and power spectrum of a mean-field of IF neurons with inhomogeneous inputs
}

\author{
Andre DH Peterson ${ }^{1,2^{*}}$, Hamish Meffin ${ }^{4}$, Anthony N Burkitt ${ }^{1,2}$, Iven MY Mareels ${ }^{1}$, David B Grayden ${ }^{1,2}$, \\ Levin Kuhlmann", Mark J Cook ${ }^{2,3}$ \\ From Nineteenth Annual Computational Neuroscience Meeting: CNS*2010 \\ San Antonio, TX, USA. 24-30 July 2010
}

The aim of this study is to construct a bottom-up model of cortical dynamics that is capable of describing the same types of neural phenomena as top-down continuum models, namely the power spectrum, frequency response to perturbation and EEG time-series. The key difference between the two approaches is that the bottom-up approach preserves more of the intrinsic physiological details than the top-down models [1]. A stochastic Fokker-Planck modelling approach is used to describe a network of leak integrate-and-fire (IF) neurons with temporally inhomogeneous inputs. Previous work either calculated the response of a single neuron with conductance-based synapses, or the network with current-based synapses [2]. In this study we use and extend a recently published Fokker-Planck approach [3] within an analytical framework to calculate the dynamical firing-rate of a network with conductance-based synapses receiving temporally inhomogeneous synaptic input. In particular, the network has fully recurrent connectivity with both the steady-state and the dynamic perturbation response of the background activity fed back into the inputs. This is done in a self-consistent formalism [4] for a network of excitatory and inhibitory neurons.

The Fokker-Planck formalism enables the calculation of the linear response of the firing-rate to perturbation with recurrent connections. The power spectrum and EEG time-series of the network are calculated by treating the synaptic inputs as an inhomogeneous Poisson process. From this we determine the auto-correlation function, which is identified as a cyclo-stationary

* Correspondence: apeterson@bionicear.org

${ }^{1}$ Department of Electrical \& Electronic Engineering, The University of Melbourne, Victoria, 3010, Australia process. The signal is then phase-averaged over its period and the Wiener-Khinchin theorem is used to determine the power spectrum from the autocorrelation function. The power spectrum is convolved with a filter to approximate the local field potential propagation through the extra-cellular fluid [5].

The analytical results of the frequency response of the dynamical firing rate and its power spectra are compared with numerical simulation results for a recurrently connected network with conductance-based synapses and temporally inhomogeneous inputs. Results are obtained using parameter values that represent typical cortical in vivo neurons [4]. This work is the first stage necessary for constructing a physiologically plausible mathematical model of a mesoscopic network of cortical columns.

\section{Acknowledgments}

This work was funded by the Australian Research Council (ARC Linkage Project \#LP0560684).

\section{Author details}

'Department of Electrical \& Electronic Engineering, The University of Melbourne, Victoria, 3010, Australia. ${ }^{2}$ The Bionic Ear Institute, 384-388 Albert St, East Melbourne, VIC 3002, Australia. ${ }^{3}$ Department of Clinical Neurosciences, St. Vincent's Hospital, Melbourne, VIC, 3065, Australia. ${ }^{4}$ NICTA VRL, C/- Dept of Electrical \& Electronic Engineering, University of Melbourne, VIC 3010, Australia.

\section{Published: 20 July 2010}

\section{References}

1. Suffczynski P, Wendling F, Bellanger JJ, Da Silva FHL: Some insights into computational models of (patho) physiological brain activity. Proceedings of the IEEE 2006, 94(4):784-804

2. Burkitt AN: A review of the integrate-and-fire neuron model: II. Inhomogeneous synaptic input and network properties. Biol Cybern 2006, 95:97-112. 
3. Richardson MJE: Spike-train spectra and network response functions for non-linear integrate-and-fire neurons. Biological Cybernetics 2008, 99(4):381-392.

4. Brunel N: Dynamics of sparsely connected networks of excitatory and inhibitory spiking neurons. J Comput Neurosci 2000, 8:183-208.

5. Bedard C, Destexhe A: Macroscopic models of local field potentials and the apparent 1/f noise in brain activity. Biophys J 2009, 96:2589-2603.

doi:10.1186/1471-2202-11-S1-P44

Cite this article as: Peterson et al.: The perturbation response and power spectrum of a mean-field of IF neurons with inhomogeneous inputs. BMC Neuroscience 2010 11(Suppl 1):P44.

Submit your next manuscript to BioMed Central and take full advantage of:

- Convenient online submission

- Thorough peer review

- No space constraints or color figure charges

- Immediate publication on acceptance

- Inclusion in PubMed, CAS, Scopus and Google Scholar

- Research which is freely available for redistribution

Submit your manuscript at www.biomedcentral.com/submit
Ciomed Central 